MAGNETIC AND LATTICE PROPERTIES OF CEBi

$\mathrm{b} \because$

G. H. Lander, M. ․ Mueller, and 0. Vogt

\begin{tabular}{|c|}
\hline 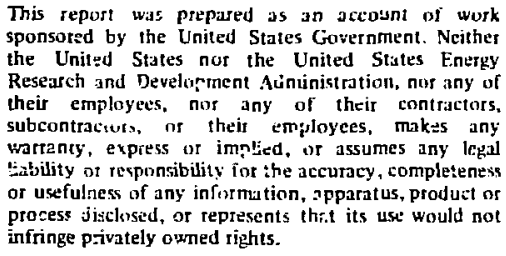 \\
\hline
\end{tabular}

Presented At:

20th Annual Conference on Magnetisn and Magnetic Materia1s, San Francisco, California, December 3-6, 1974 
MAGNETIC AND LATTICE PROPERTIES OF C.EBi

\author{
G.H. Lander, M.H. Mueller, \\ Argonne National Laboratory, Argonne Illinois 60439 \\ 0. Vogt \\ Laboratory für Festkorperphysik, ETH, Zurich, Switzerland
}

\begin{abstract}
The magnetic structures of CeBi have been examined as a function of field with neutron diffraction. At low fields unusual domain reorientation effects are observed. For $\mathrm{H}>18 \mathrm{k0e}$ a $3+, 1-$ spln configuration is found. A first-order transition to induced ferromagnetic behavior occurs at $47.5 \mathrm{k0e}$. X-ray experiments at low temperature show that no lattice distortion occurs at either the NEel temperature or the I - IA transition at $12.5 \mathrm{~K}$. A volume discontinuity is observed at the low temperature transition.
\end{abstract}

\title{
INTRODUCTION
}

The compound $\mathrm{CeBi}$ (NaCl structure) is of interest because of its anomalous magnetization 1,2 and unusual magnetic structures in zero field. ${ }^{3}$ At $T_{N}(25 \mathrm{~K})$ the magnetic structure is type $I$ in which ferromagnetic (001) sheets are stacked in an alternating +,sequence. The spin direction is parallel to [001]. However, at $12.5 \mathrm{~K} \mathrm{CeBi}$ undergoes a first-order transition to the type IA magnetic structure, In which the spin direction remains unaltered but the ferromagnetic sheets are stacked in the +t- sequence. The if structure is unusual in that it is not predicted by molecular field theory, but has been observed in a number of uranium compounds. ${ }^{4}$ Recently Cooper et al. ${ }^{5}$ and Bartholin ${ }^{6}$ have reported magnetization experiments on single crystals. These measurements have shown that the moments are parallel to the cube axis in all fields and that for $15<\mathrm{H}<45 \mathrm{k0e}$ ar intermediate phase exists in which the magnetization is $1 / 2$ the value in the fully ordered state $(=2.1 \mu \mathrm{H} / \mathrm{Ce}$ atom).

\section{Neutron experiments}

The experiments were performed at the $\mathrm{CP}-5$ Research Reactor with a $60 \mathrm{kne}$ superconduct Ing magnet assembly. The fileld was applied parallel to [001] throughout. The following reflectlons (together with their interpretation) were examined as a function 
of field: (110) - type I modulation with the propagation direction $\mathrm{T} \| \mathrm{H},(210)$ - type 1 modulation $\mathrm{T} \perp \mathrm{H}$, (11 $1 / 2)$ - type IA modulation T $\| \mathrm{H},(21 / 20)$ - type IA modulation $\tau \perp \mathrm{H}$, and the (111) and (200) nuclear reflections which are sensitive to a net forromagnetic contribution and are equivalent to the magnetiantion. For the (111) reflection the nuclear contribution is $\mathrm{N}(111)=\mathrm{b}_{\mathrm{Ce}}-\mathrm{b}_{\mathrm{Bi}}=-0.377 \times 10^{-12} \mathrm{~cm}$, and the magnet $\mathrm{c}$ contribution $M(111)=0.27 \times \mathrm{f} \times \mu$, where $f$ is the form factor $(=0.91)$ from Blume et $a .^{7}$ and the dipole approximation. ${ }^{8}$ Thus $M(111)=0.245 \times \mu$, where $\mu$ is the moment per $C e$ in Bohr magnetons. The polarized-neutron technique measures the ratio $\mathrm{M} / \mathrm{N}$ so the (111) reflection is very sensitive to a ferromagnetic component. a) Type in phase, $\mathrm{T}=5 \mathrm{~K}$.

Figure 1 (left hand side) shows the field dependence of the (110) and $(111 / 2)$ reflections together with the ferromagnetic component. Initially with $\mathrm{H}=0$ only the rype IA reflections are present. As $H$ increases the (11 $1 / 2$ ) increases and the $(21 / 20)$ decreases (not shown). This behavior corresponds to a domain reorientation such that preferred domains have $\tau \| \mathrm{H}, \mathrm{i} . e$. the longltudinal susceptibllity is greater than the transverse, and was observed by Cable and Koehier. ${ }^{3}$ For $\mathrm{H} \sim 15 \mathrm{k0e}$ we belleve fluctuations in the arrangement of the ferromagnetic (001) sheets leads to a lack of long-range coherence and the resulting loss of antiferromagnetic intensity. A sharp rise in the ferromagnetic component occurs at this field. For $19<H<47.5$ ?0e both the $(110)$ and $(111 / 2)$ reflections are present, $a=$ well as a ferromagnet1c component of $1.05 \mu_{\mathrm{B}} / \mathrm{C} \in$ atom. $\mathrm{M}_{0}$ intensity was observed at the (210) or $(21 / 20)$ positions. A combination of these modulations leads to the conclusion that the spin configuration is a $3+, 1$ - sequence of the ferromagnetic (001) sheets. For $\mathrm{H}=47.5 \mathrm{kOe}$ the 3+, 1- structure collapses, leading to a totaliy induced ferromagnetic state with $2.1 \mu_{B} / \mathrm{Ce}$ atom.

b) Type I phase, $12.5<\mathrm{T}<25 \mathrm{~K}$.

Figure 1 (right hand side) shows the field dependence of the (110) and $(111 / 2)$ reflections, together with the ferromagnetic component at $14 \mathrm{~K}$. In contrast to the low temperature behavior, the transverse susceptibility in the type I phase is greater than the longitudina] (as one expects for an antiferromagnet) and the (110) decreases as $H$ increases. For $H$ between 10 and $17 \mathrm{kle}$ the behavior is complex, and, we belleve, represents both the domain reorientation effects and the inherent instability of the I and IA structures in the presence of an applied field. This behavfor extends over a wider field range as the temperature is increased towards $T_{N}(25 \mathrm{~K})$. The lack of any magnetic intensity at the (210) or ( $\angle 1 / 20)$ posf.tions eliminates the possibility of a canted spin arrangement. For 
$17.5<\mathrm{H}<47.5 \mathrm{kOe}$ the $3+, 1-$ configuration is again present. The critical field for induced ferromagnetism shows 1ittle temperature dependence for $\mathrm{T}<20 \mathrm{~K}$, in agreement with magnetization results.

\section{X-ray experiments}

We have examined the behavior of the lattice parameter of $\mathrm{CeBi}$ at low temperature with X-ray diffraction from the ( 800$)$ planes of a single crystal. The results are shown in Fig.2. A careful search for a lattice distortion in the ordered state gave an upper limit of $|(c-a) / a|<1 \times 10^{-4}$ for any tetragonal distortion. As the figure shows, on warming through the IA-I transition (12.5K) the relative volume discontinuity associated with the first-order transition is $(8 \pm 1) \times 10^{-5}$. The thermal expansion in the type I magnetic phase is first positive $(T<20 \mathrm{~K})$ then negative for $\mathrm{T}>20 \mathrm{~K}$. The lattice parameter at $5 \mathrm{~K}$ is 6.4873 (1) $\AA$. The change in the lattice parameter between 25 and $30 \mathrm{~K}$ represents a linear expansion coefficient of $7 \times 10^{-6} /{ }^{\circ} \mathrm{K}$.

\section{DISCUSSION}

The experiments reported here confirm the $3+, 1-$ configuration for the intermediate field state in CeBi as first suggested by Tsuchida and Nakamura, ${ }^{9}$ In fact their phase diagram is in excellent agreement with our results and we present a modified phase diagram with all the magnetic structures defined in Fig. 3. The dashed 1ines represent boundaries that have not been experimentally verified. The variations in the intensities of the (110) and $(11$ 1/2) at low fields (see Fig.1) arise from domain reorientation effects and/or the instability of the I and IA phases to the application of a magnetic field and, as such, are not represented in the phase diagram. For $\mathrm{H}>47.5 \mathrm{k0e}$ and $\mathrm{T}<25 \mathrm{~K}$ the correct description of the phase is paramagnetic rather than the "induced ferromagnetism" used in the 1iterature. ${ }^{9}$ However, the anisotropy in this state is considerable, and the spins cainnot be rotated significantly away from the cube axis.5,6 As discussed by Cooper et al. ${ }^{5}$ the easy direction for the mements in CeBi should be $\langle 111\rangle$ if the crystal field is the dominant interaction. Direct measurements of the crystal-field splittings in CeBi have found them to be smallio se that it is more likely that the exchange interactions define the easy axis of magnetization. 
The mechantsm that stabilizes the IA magnetic structure remains a mystery. Particularly since no distortion, which would allow the introduction of an additional term in the free energy, is observed at the I - IA transition at $12.5 \mathrm{~K}$. The close analogy between the uranium compounds with the I and TA structures ${ }^{4}$ (and their unusual behavior in a magnetic field) and CeB1 is even more striking when one recalls that these urantum compounds, like CeBi, maintain cubic symmetry below their respective ordering temperatures. 11

\section{REFERENCES}

1. Y. L. Wang and B. R. Cooper, Phys. Rev. B 2,2607 (1970), and references therein.

2. T. Tsuchida, A. Hashimoto, and Y. Nakamura, J. Phys. Soc. Japan 36, 685 (1974)

3. J. W. Cable and W. C. Koehler, AIP Conf. Proc. 5, 1381 (1972)

4. G. H. Lander, M. H. Mueller, and J. F. Reddy, Phys. Rev. B 6 , 1880 (1972); R. C. Maglic, G. H. Lander, M. H. Mueller, J. Crangle. and G. S. Williams, Phys. Rev. B 10, 1943 (1974)

5. B. R. Cooper, $M$. Landolt, and $n$. Vogt, Int. Cont. on Magnetism, Moscow, Aug. 1973, paper 27a-s4.

6. H. Bartholin, private communication.

T. M. Blume, A. J. Freeman, and R. F. Watson, J. Chem. Phys. 37, 1245 (1962)

8. F. H. Lander and T. O. Rrun, ibtd 53, 1387 (1970)

9. T. Tsuchida and Y. Nakamura, J. Phys. Soc. Japan 22, 942 (1967)

10. A. Furrer, W. Buhrer, H. Heer, W. Halg, J. Benes, and 0 . Vogt. Neutron Inelastic Scattering 1972 (IAFA, Vienna), 563,1972

11. F. H. Lander and M. H. Mueller, Phys. Rev. B 10, 1994 (1974) 

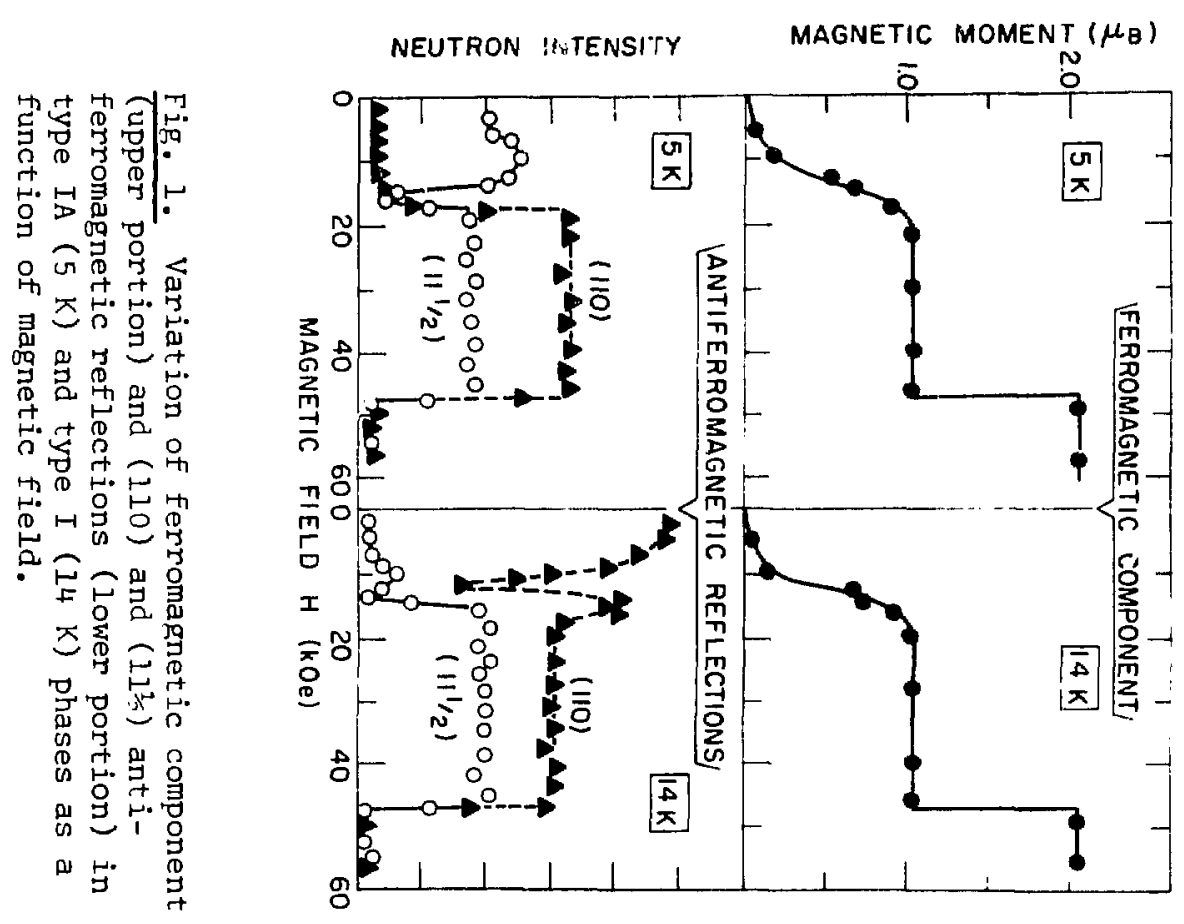


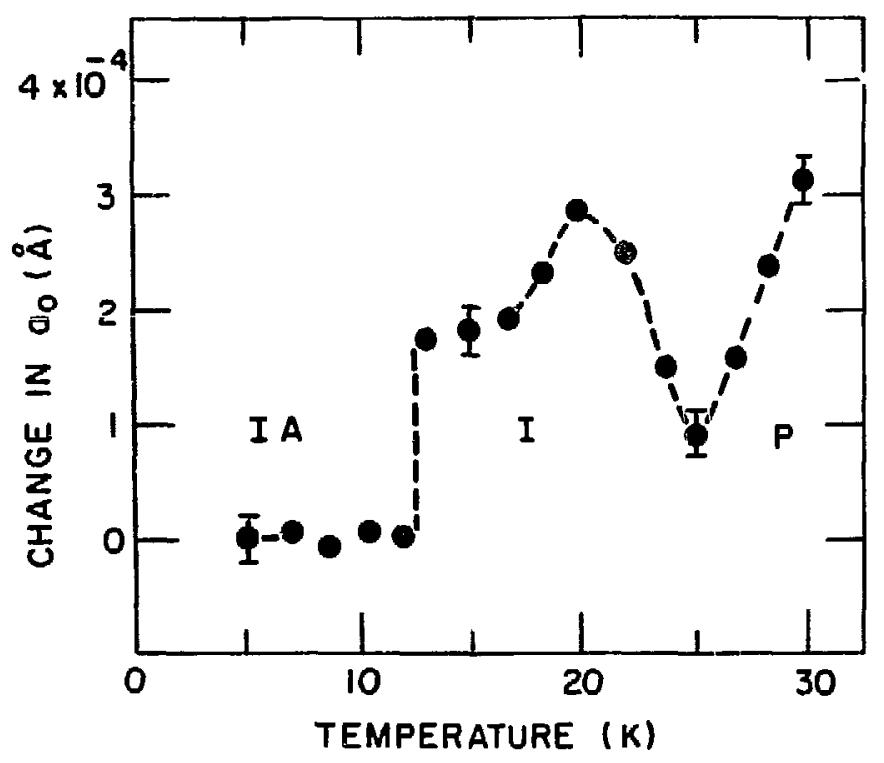

Fig. 2. Change of lattice prarameter Trelative tc value of $\epsilon .487 \AA \AA$ at $5 \mathrm{k}$ ) of $\mathrm{CeBi}$ as a function of temperature. 


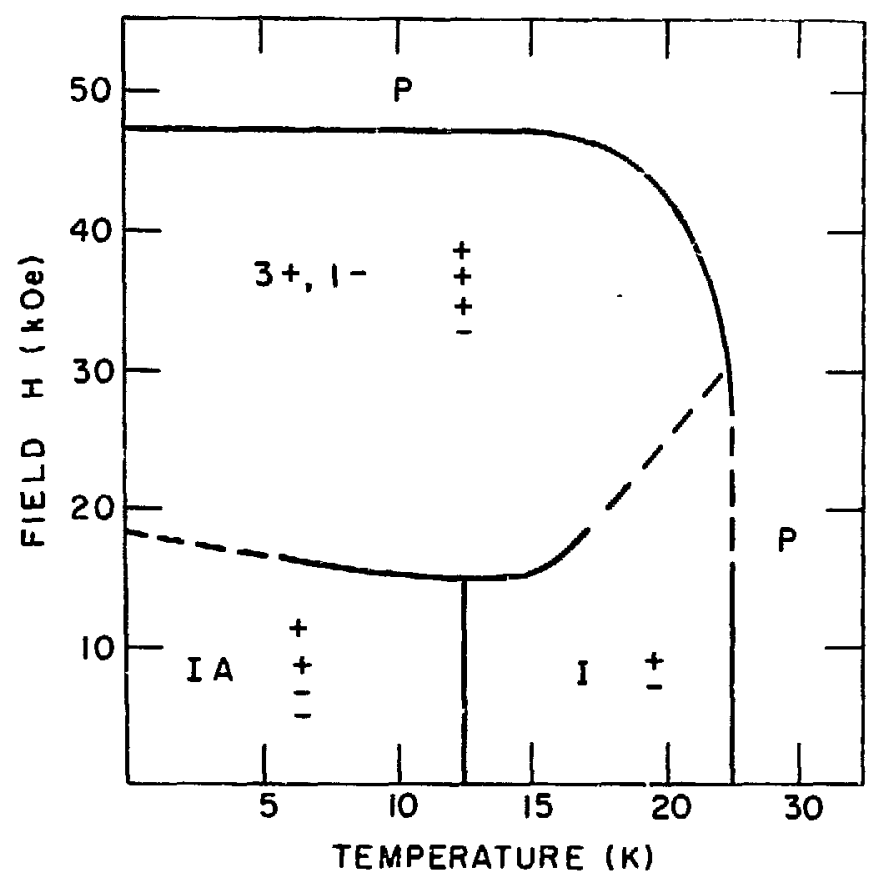

Fig. 3. Phase diagram for CeBi. 